\title{
Implante coclear para el manejo del tinnitus intratable en pacientes con hipoacusia unilateral
}

\section{Cochlear implant for the management of intractable tinnitus in patients with unilateral hearing loss}

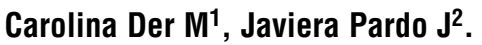

\begin{abstract}
RESUMEN
El tinnitus es una condición frecuente en la población y altamente invalidante. Se han aplicado numerosas modalidades de tratamiento, sin embargo aún no hay consenso sobre un agente terapéutico efectivo. Incidentalmente se ha visto que los pacientes con hipoacusia profunda bilateral y tinnitus que han recibido un implante coclear, refieren mejoría de este síntoma en un alto porcentaje. Esto ha llevado a la indicación del implante coclear en casos de tinnitus intratable. Estudios recientes, han demostrado que el uso de esta audioprótesis aparece como una alternativa prometedora para el manejo del tinnitus en pacientes con sordera unilateral.
\end{abstract}

Palabras clave: Tinnitus, implante coclear, hipoacusia unilateral.

\begin{abstract}
Tinnitus is a common and highly disabling condition in the population. Many forms of treatments have been implemented. However, there is no consensus yet on an effective therapeutic agent. Incidentally, it has been observed that patients with bilateral profound hearing loss and tinnitus that have received a cochlear implant, report improvement of this symptom in a high percentage. This has led to the indication of cochlear implant in intractable tinnitus cases. Recent studies have shown that using this hearing aid appears as a promising alternative for the management of tinnitus in patients with unilateral deafness.
\end{abstract}

Key words: Tinnitus, cochlear implant, unilateral hearing loss.

Médico. Servicio de Otorrinolaringología, Hospital Clínico Universidad de Chile y Clínica Alemana de Santiago

2 Médico Cirujano. Tesista de Magíster en Ciencias Biomédicas, mención Neurociencias, Universidad de Chile. 


\section{INTRODUCCIÓN}

Se define tinnitus o acúfenos como la percepción de sonido en ausencia de un correlato acústico 0 mecánico en la cóclea ${ }^{1}$. Representa un síntoma y no una enfermedad. Es considerado uno de los problemas otológicos más comunes e invalidantes, ya que genera molestias tanto somáticas como psicológicas que interfieren con la calidad de vida de los pacientes. Su prevalencia se incrementa con la edad².

Dado que no es una enfermedad en sí misma, sino que es un síntoma que caracteriza a una gran variedad de patologías, se identifican múltiples causas que lo generan. Dentro de las causas otológicas se incluye presbiacusia, hipoacusia inducida por ruido, otoesclerosis, otitis de diversa etiología, cerumen impactado, sordera súbita, enfermedad de Ménière y otras causas de pérdida auditiva.

Además se presenta asociado a patologías neurológicas como daño cerebral, esclerosis múltiple o schwannomas vestibulares y otros tumores del ángulo pontocerebeloso. Procesos infecciosos como secuelas de enfermedad de Lyme, meningitis y sífilis también pueden provocarlo.

Ocurre también como efecto adverso del uso de algunos medicamentos ototóxicos, como salicilatos, antiinflamatorios no esteroidales, aminoglicósidos, diuréticos de asa y agentes quimioterapeúticos como cisplatino y vincristina.

La disfunción temporomandibular y otros problemas dentales también pueden causar tinnitus. Sin embargo, en muchos casos no existe una causa física identificable ${ }^{3}$.

Se han propuesto muchas teorías para explicar el mecanismo subyacente a este fenómeno. Lo que hoy sabemos con certeza es que el evento más frecuentemente asociado al tinnitus es la hipoacusia.

\section{EPIDEMIOLOGÍA, FISIOPATOLOGÍA Y MECANISMOS PROPUESTOS}

El tinnitus es una condición muy frecuente en la población, estimándose su prevalencia entre $8,2 \%$ y $30,3 \%$ en ciudades industrializadas ${ }^{4}$. En pacientes candidatos a implante coclear la prevalencia fluctúa entre $66 \%$ y $86 \%$ preimplantación.
El conocimiento actual sobre la fisiopatología del acúfenos es aún incompleto, sin embargo existe evidencia creciente indicando que estaría relacionado con alteraciones en el funcionamiento neuronal del sistema auditivo central.

Similar a lo que ocurre con el dolor del miembro fantasma, el tinnitus se interpretaría como una percepción auditiva fantasma, la que se correlaciona con intentos de adaptación del cerebro frente a los inputs sensoriales distorsionados generados por un oído con función auditiva deficiente. Este concepto se ve reforzado por el antecedente de que la pérdida auditiva es el factor de riesgo más importante para desarrollar acúfenos ${ }^{5}$.

Experimentos en animales han demostrado que al reducir los input auditivos se produce un desbalance entre mecanismos inhibitorios $y$ facilitadores en toda la vía auditiva central, que genera como resultado reorganización de los mapas tonotópicos en la corteza auditiva. Esto representa el correlato neuronal del tinnitus ${ }^{6}$.

La visión tradicional plantea que ante una lesión coclear se produce la generación de acúfenos. Sin embargo, múltiples estudios proponen que existen muchos otros mecanismos involucrados ${ }^{7,8}$.

Mediante estudio de imágenes con PET, se ha logrado mapear los sitios de actividad cerebral en sujetos que modulan la intensidad de su tinnitus con movimientos orofaciales, en sujetos que evocan tinnitus mediante mirada fija y en sujetos en que se aumenta 0 disminuye la intensidad del tinnitus en respuesta a lidocaína endovenosa. Estos estudios muestran que el acúfenos surge de la hiperactividad del sistema auditivo cerebral central cuando disminuye el input proveniente de la cóclea. Esta hiperactividad es percibida como acúfenos. Drogas como la lidocaína reducen el tinnitus, mediante la disminución del flujo cerebral en la corteza de asociación derecha? ${ }^{9}$.

\section{MECANISMOS DE ENMASCARAMIENTO SONORO E INHIBICIÓN RESIDUAL}

Una de las propiedades fundamentales del tinnitus es que puede ser enmascarado por sonidos externos ${ }^{10}$. La reducción del tinnitus que se produce 
luego de la cesación del enmascaramiento, se conoce como inhibición residual. Los mecanismos tras este fenómeno son similares y se sobreponen a la génesis del acúfenos ${ }^{11}$. Diferentes estudios sugieren que muchas formas de tinnitus resultan de una pérdida de la inhibición de estructuras auditivas centrales secundario a pérdida auditiva o procesos de envejecimiento. Esto genera actividad neural sincrónica entre las redes neuronales de la corteza auditiva, dando lugar a la sensación de acúfenos ${ }^{12}$.

La intensidad de sonido necesaria para enmascarar el acúfenos es menor si coincide la frecuencia de enmascaramiento con la frecuencia de la región auditiva con déficit. De esta forma, el espectro de frecuencia del tinnitus se sobrepone con el espectro de frecuencia de la pérdida auditiva.

Por otra parte, los enmascarantes sólo son efectivos si son presentados sobre los umbrales de sonido individuales ${ }^{13}$. Cuando el enmascaramiento, de intensidad suficiente, es presentado en la región de la disminución auditiva, la excitación es enviada a la corteza a través de las vías talamocorticales, lo que produce una gran inhibición, luego de un retraso sináptico ${ }^{14}$. El código de propiedades acústicas contenidas en el sonido enmascarante genera la percepción normal, que interfiere con la percepción del tinnitus. De esta forma, sonidos presentados en otras frecuencias (fuera de las frecuencias de la región cortical afectada por la hipoacusia) mantiene el acúfenos intacto. Sin embargo, si el enmascaramiento se produce fuera de las frecuencias, pero a gran intensidad el acúfenos también es suprimido $0^{10}$. Esto debido a que las neuronas de la corteza auditiva pierden la especificidad por frecuencia con sonidos de alta intensidad ${ }^{15}$. De esta forma la inhibición residual representaría la segregación temporal de la sincronía neural anormal, que persiste más allá del enmascaramiento.

La inhibición residual requiere de sonidos enmascarantes que excedan el nivel de enmascaramiento mínimo ${ }^{11}$. La profundidad y duración de la inhibición residual aumenta si la intensidad del enmascaramiento supera $20 \mathrm{~dB}$ sobre el nivel mínimo. Esta relación dosis respuesta es consistente con la disrupción de la sincronía neural como base del mecanismo.

La duración del enmascaramiento es importante. Así, la duración de la inhibición residual aumenta linealmente en función del logaritmo de la duración del enmascarante, para duraciones entre 10 segundos y 10 minutos $^{16}$. Los factores que predicen este fenómeno son desconocidos. Por otra parte, el enmascaramiento es superior cuando el estímulo es presentado en forma independiente en cada oído (estimulación dicótica), que cuando el mismo sonido es presentado simultáneamente en ambos oídos (estimulación diótica) ${ }^{17}$.

La aplicación clínica de la inhibición residual tiene como limitante práctica la intensidad del sonido. Para pacientes con hipoacusia severa, los enmascarantes deben ser presentados a intensidades necesarias para inducir inhibición residual (10 $\mathrm{dB}$ sobre el nivel de enmascaramiento mínimo), que entrega una presión de sonido sobre $100 \mathrm{~dB}$ en cada oído. Su continua exposición puede exacerbar la lesión auditiva periférica. La estimulación magnética transcraneal tiene la ventaja de producir el mismo efecto sin requerir la vía auditiva para llegar al cerebro, ya que alcanza directamente las áreas cerebrales seleccionadas ${ }^{11}$.

\section{TRATAMIENTOS}

La remisión espontánea por habituación es la evolución natural del tinnitus en el $75 \%$ de los $\operatorname{casos}^{18}$. En los casos en que esto no ocurre y el acúfenos persiste por más de 2 años, sin respuesta a algún tratamiento previo, se considera permanente e irreversible. Esto generalmente asociado a un puntaje de la escala Tinnitus Handicap Inventory (THI) en rango de severo o catastrófico ${ }^{19}$.

Numerosas modalidades de tratamiento han sido aplicadas, sin embargo aún no hay consenso sobre la emergencia de un agente terapéutico efectivo. Hasta ahora no existe un tratamiento que represente la mejor alternativa. La complejidad de los cambios en el sistema nervioso asociados al tinnitus explicaría la resistencia al tratamiento ${ }^{3}$.

Entre las terapias disponibles se cuenta con fármacos, terapia cognitiva conductual, terapia de sonido, habituación, masajes, uso de audífonos y estimulación eléctrica ${ }^{20}$.

En relación a los fármacos, los que han mostrado un beneficio mayor que placebo son nortriptilina, amitriptilina, alprazolam, clonazepam y oxacepam $^{21,22}$. Sin embargo, al descontinuar el uso de éstos, el acúfenos reaparece. 
El único fármaco que ha demostrado reducción importante del tinnitus es la lidocaína endovenosa. Ésta produce cambios en la actividad neural del lóbulo temporal derecho en la corteza de asociación auditiva ${ }^{9}$. Lamentablemente no puede ser utilizada clínicamente, ya que debe ser inyectada, su efecto es de corta duración y no está exenta de efectos adversos.

La terapia cognitiva conductual se basa en evitar la ideación negativa sobre el acúfenos para lo cual se utiliza la desensibilización sistemática tal como se aplicaría en pacientes fóbicos.

En cuanto al uso de estimulación sonora ésta ha mostrado algunos resultados positivos sólo durante el período de aplicación.

\section{ELECTROESTIMULACIÓN}

La estimulación eléctrica en la cóclea puede suprimir el acúfenos en algunos pacientes. La estimulación unilateral de la cóclea normalmente produce reducción del tinnitus monoaural, pero algunos pacientes reportan disminución del tinnitus biaural. Es posible suprimir el acúfenos sin percibir la estimulación ${ }^{23}$.

En algunos pacientes el tinnitus puede ser disminuido por minutos, incluso horas después de apagado el estímulo eléctrico. Este fenómeno se denomina efecto residual ${ }^{24}$.

\section{TERAPIA ELECTROMAGNÉTICA}

El acúfenos frecuentemente se asocia a sordera, pudiendo ser el resultado de un proceso de plasticidad patológica ${ }^{25}$. Estudios de neuroimágenes han demostrado hiperactividad del sistema auditivo central. La estimulación magnética transcraneal repetitiva (EMTr) es un método relativamente nuevo, no invasivo, que modula la excitabilidad de la corteza cerebral. Su uso ha demostrado ser efectivo en el manejo de patologías neuropsiquiátricas, como alucinaciones auditivas, entre otras ${ }^{26}$. Su uso guiado mediante neuronavegación sobre el área de actividad aumentada ha demostrado reducir la percepción de tinnitus ${ }^{27}$. Esta estimulación es realizada en forma altamente selectiva y estandarizada. El estudio realizado por
Langgut y cols a 28 pacientes con tinnitus crónico intratable mostró mejoría significativa del acúfenos en 19 pacientes, medido con un cuestionario estandarizado, luego de 10 sesiones. Una de las hipótesis para explicar este fenómeno, es que la estimulación magnética transcraneal repetitiva de baja frecuencia, ejerce un efecto terapeútico a través de la depotenciación selectiva de las sinapsis potenciadas patológicamente ${ }^{28}$. Otra explicación es que su aplicación modula los circuitos neuronales ${ }^{29}$. Pese a todo el conocimiento actual, se necesitan estudios controlados con placebo.

\section{IMPLANTE COCLEAR Y TINNITUS}

La indicación clásica de implante coclear es la hipoacusia severa 0 profunda bilateral. La prevalencia de tinnitus en pacientes adultos con indicación de implante coclear fluctúa entre $67 \%$ y $100 \%{ }^{30}$.

Los efectos beneficiosos del implante coclear sobre el acúfenos han sido demostrados en la literatura por varios autores ${ }^{31,32}$. El implante coclear unilateral es capaz incluso de beneficiar a pacientes con tinnitus bilaterales. Se describe desaparición del tinnitus tanto ipsi como contralateral en un porcentaje mayor al $50 \%$ previo al encendido del implante, lo que aumenta aún más cuando éste es encendido ${ }^{33}$.

Se realizó un estudio a 41 pacientes con sordera profunda. Se les implantó un dispositivo multicanal para evaluar el efecto del implante sobre el acúfenos bilateral. Se demostró que el tinnitus fue abolido en el $56,1 \%$ de los pacientes con el implante apagado en el oído implantado y en $53,6 \%$ en el oído contralateral. Con el implante encendido, la abolición del acúfenos fue de $65,8 \%$ tanto en el oído ipsilateral como contralateral, esto medido por un cuestionario de evaluación de la intensidad del tinnitus. Además se observó reducción en el puntaje de la escala Tinnitus Handicap Inventory (THI) ${ }^{33}$.

El efecto beneficioso tanto en el oído ipsilateral, como en el contralateral estaría determinado según Battmer, por el efecto de enmascaramiento acústico mediante estimulación eléctrica. Sin embargo, la teoría del enmascaramiento acústico no explica la supresión del tinnitus cuando el implante se encuentra apagado. 
En relación a esto se ha postulado que la colocación del implante coclear genera trauma inmediato y posterior sobre las estructuras cocleares restantes. Esto podría ser una ventaja en los pacientes, en los cuales la actividad anormal de las células ciliadas es el mecanismo gatillante del acúfenos, por lo que se esperan efectos inmediatos posoperatorios independiente de la activación del implante. Este mecanismo explicaría la atenuación 0 abolición del tinnitus con el implante apagado ${ }^{34}$.

Si se considera el modelo fisiopatológico del acúfenos como una percepción auditiva fantasma que se correlaciona con intentos maladaptativos de reorganización cortical secundarios a la falta de aferencias periféricas, el restaurar los input sensoriales genera efectos benéficos a largo plazo sobre el tinnitus mediante reorganización plástica del sistema nervioso auditivo central, lo que se correlaciona con los hallazgos clínicos encontrados por Kleinjung, con mejoría del acúfenos a los tres meses posimplante ${ }^{34,35}$.

Al analizar los diferentes tipos de implantes cocleares no existen diferencias significativas, en lo que al modelo de implante se refiere. Entre las 5 estrategias usadas en el estudio de Quaranta (SPEAK, ACE, SAS, CIS y HR) no parece que alguna de ellas sea superior a las otras con el implante apagado. Con el implante encendido, estrategias más sofisticadas y con codificación rápida del lenguaje, como ACE, HR y CIS generan una mayor supresión del tinnitus comparado con estrategias como SPEAK y SAS ${ }^{33}$.

Existe un estudio que muestra la aplicación de implantes cocleares para el manejo del acúfenos en pacientes con sordera unilatera ${ }^{36}$. Los pacientes incluidos en este estudio tenían hipoacusia unilateral acompañada de tinnitus severo durante los últimos dos años. En el $95 \%$ de los pacientes se demostraron efectos beneficiosos: en tres pacientes el tinnitus cedió completamente y en la mayoría de los pacientes se demostró mejoría estadísticamente significativa de la intensidad e impacto del acúfenos $^{36}$.

Se ha descrito empeoramiento del tinnitus preexistente o desarrollo de un nuevo tinnitus tras la implantación ${ }^{24}$. El riesgo de desarrollar acúfenos después del implante coclear varía entre $0 \%$ y $4 \%{ }^{37-40}$. El empeoramiento del tinnitus preexistente se ha reportado entre $1 \%$ y $9 \%$ de los $\operatorname{casos}^{38,41-43}$.

\section{AUDICIÓN BIAURAL Y DISCRIMINACIÓN}

Los individuos que desarrollan una hipoacusia sensorioneural unilateral se dan cuenta de la importancia de la audición biaural en la vida diaria. Presentan dificultades en seguir conversaciones, incapacidad de localizar el sonido y dificultades para entender conversaciones en ambientes ruido$\mathrm{SOS}^{44}$.

Históricamente esta desventaja ha sido subestimada, sin embargo la superioridad de la audición biaural por sobre la monoaural ha sido bien establecida en la literatura ${ }^{45-48}$.

La audición biaural no sólo provee sumación de intensidad sonora, sino que también evita el efecto de sombra acústica generado por la cabeza mejorando la discriminación del lenguaje en ambientes ruidosos, así como la orientación espacial y localización del sonido ${ }^{49}$.

Muchos de los problemas en pacientes con audición unilateral se presentan en relación al efecto de sombra de la cabeza. En los casos en que la cabeza se encuentra entre la fuente sonora y el oído con audición normal, se genera atenuación de la intensidad de la señal en el lado opuesto a la cabeza. En este caso la cabeza actúa como una barrera acústica al sonido, por lo que el sonido y el ruido provenientes de diferentes localizaciones espaciales llegan a dicho oído con diferentes intensidades. El efecto de sombra de la cabeza es más pronunciado a altas frecuencias ${ }^{50}$.

El oído que se encuentra más cercano a la fuente de ruido tendrá una proporción de señal/ ruido inferior comparado con el oído ubicado más cerca de la señal de lenguaje, el que tendrá una ventaja auditiva.

La segunda ventaja de la audición biaural sobre la comprensión del lenguaje se genera desde el procesamiento de la señal cerebral proveniente de inputs de ambos oídos. Cuando el input proviene de ambos lados el cerebro realiza una mejor representación del sonido y el lenguaje. Cuando el lenguaje y el ruido provienen de localizaciones diferentes, el cerebro es capaz de separarlos usando distintos tiempos interaurales, nivel y claves espectrales que mejoran la discriminación. Esto se denomina binaural squelch ${ }^{45,51}$.

En un sujeto normal el efecto squelch genera una ganancia de $2 \mathrm{~dB}$ a $4,9 \mathrm{~dB}$ en el umbral de 
percepción del lenguaje ${ }^{45,47,48,52}$. La combinación de efecto de sombra de la cabeza y el efecto squelch mejora el reconocimiento de monosílabos en más de $40 \%$ en sujetos con audición normal ${ }^{53}$.

La tercera ventaja relacionada a la comprensión del lenguaje y el efecto de sumación biaural, se produce cuando señales idénticas llegan a ambos oídos. La información redundante recibida por ambos oídos produce una mejoría en la percepción del lenguaje. Este efecto genera una pequeña mejoría de $1 \mathrm{~dB}$ a $2 \mathrm{~dB}$ en el umbral de percepción del lenguaje en sujetos con audición normal ${ }^{53}$.

Dados los avances tecnológicos del implante coclear y estrategias de procesamiento del lenguaje, la selección de candidatos para implante coclear ha sido extendida a sujetos con hipoacusia moderada a severa. En relación a esto ha aumentado el número de pacientes con implante unilateral y audición residual en el oído no implantado, en el que utilizan audífono. En estos pacientes se ha observado efecto de sumación biaural ${ }^{54}$, el que también se ha observado en pacientes usuarios de audífonos bilaterales ${ }^{5,56}$.

En 2009 Vermeire publicó los resultados auditivos de 20 pacientes implantados con hipoacusia unilateral y tinnitus intratable en el oído operado. Se observó mejoría significativa en la configuración espacial así como un importante beneficio global en la calidad de vida ${ }^{56}$.

\section{CONCLUSIÓN}

En la actualidad los pacientes sordos de escasos recursos de nuestro país tienen un acceso muy limitado al implante coclear. Es fundamental concentrar nuestros esfuerzos en lograr un cambio en este sentido. Sin embargo también es importante conocer hacia dónde se dirigen las nuevas indicaciones de esta audioprótesis.

Dados los excelentes resultados clínicos del implante coclear, cada vez parecen más cercanas sus aplicaciones en otras patologías auditivas, como es el caso del tinnitus intratable. Es de especial importancia la realización de nuevos estudios que permitan mejorar el nivel de evidencia al respecto. Esto nos permitirá ampliar nuestros conocimientos e indicar correctamente su uso en pacientes seleccionados.

\section{BIBLIOGRAFÍA}

1. Jastreboff PJ. Phantom auditory perception (tinnitus): mechanisms of generation and perception. Neurosci Res 1990; 8: 221-54.

2. Daniell WE, Fulton-Kehoe D, Smith-Weller T, Franklin GM. Occupational hearing loss in Washington state, 1984-1991: II. Morbidity and associated costs. Am J Ind Med 1998; 33: 529-36.

3. Lockwood AH, Salvi RJ, Burkard RF. Tinnitus. $N$ Engl J Med 2002; 347: 904-10.

4. SÁnchez L. The epidemiology of tinnitus. Audiological Medicine 2004; 2: 8-17.

5. Kleinjung T, Steffens T, Strutz J, Langguth B. Curing tinnitus with a Cochlear Implant in a patient with unilateral sudden deafness: a case report. Cases Journal 2009; 2: 7462.

6. EgGermont JJ. Pathophysiology of tinnitus. Prog Brain Res 2007; 166: 19-35.

7. Baguley DM. Mechanisms of tinnitus. Br Med Bull 2002; 63: 195-212.

8. Salvi RJ, Lockwood AJ, Burkard R. Neural plasticity and tinnitus. In: Tyler RS, editor. Tinnitus Handbook. San Diego: Singular; 2000; 123-48.

9. Reyes SA, Salvi RJ, Burkard RF, Coad ML, Wack DS, Galantowicz PJ, et al. Brain imaging of the effects of lidocaine on tinnitus. Hear Res 2002; 171: 43-50.

10. Feldman H. Homolateral and contralateral masking of tinnitus by noise-bands and by pure tones. Audiology 1971; 10: 138-44.

11. RoberTs LE. Residual inhibition. Prog Brain Res 2007; 166: 487-95.

12. EgGermont JJ, Roberts LE. The neuroscience of tinnitus. Trends Neurosci 2004; 27: 676-82.

13. Vernon JA, Meikle MB. Tinnitus: clinical measurement. Otolaryngol Clin N Am 2003; 36: 293-305.

14. Cruikshank SJ, LeWIS TJ, Connors BW. Synaptic basis for intense thalamocortical activation of feedforward inhibitory cells in neocortex. Nat Neurosci 2007; 10: 808-10.

15. Phillips DP, Semple MN, Calford MB, Kitzes LM. Level-dependent representation of stimulus frequency in cat primary auditory cortex. Exp Brain Res 1994; 102: 210-26.

16. Terry AMP, Jones DM, Davis BR, Slater R. Parametric studies of tinnitus masking and 
residual inhibition. Br J Audiol 1983; 17: 24556.

17. Johnson RM, Hughes FM. Diotic versus dichotic masking of tinnitus. In: Aran J.-M. and Dauman R. (Eds.), Proceedings of the Fourth International Tinnitus Seminar. Kugler Publications, Amsterdam, 1992; 387-90.

18. Jastreboff PJ, HazeLL JW. Tinnitus Retraining Therapy. New York: Cambridge University Press, 2004.

19. Heller AJ. Classification and epidemiology of tinnitus. Otolaryngol Clin North Am 2003; 36(2): 239-48.

20. Byung In Han, Ho Won Lee, Tae You Kim, Jun Seong LIM, Kyoung SIK ShIN. Tinnitus: Characteristics, Causes, Mechanisms, and Treatments. J Clin Neurol 2009; 5: 11-9.

21. DobIE RA. A review of randomized clinical trials in tinnitus. Laryngoscope 1999; 109: 1202-11.

22. Mural K, Tyler RS, Harker LA, Stouffer JL. Review of pharmacologic treatment of tinnitus. Am J Otol 1992; 13: 454-64.

23. Rubinstein JT, Tyler RS, Johnson A, Brown CJ. Electrical suppression of tinnitus with high-rate pulse trains. Otol Neurotol 2003; 24: 478-85.

24. Tyler RS, Rubinstein J, Pan T, Chang SA, Gogel SA, Gehringer A, Coelho C. Semin Electrical Stimulation of the Cochlea to Reduce Tinnitus. Hear 2008; 29(4): 326-32.

25. Langguth B, Hajak G, Kleinjung T, Pridmore S, Sand P, Eichhammer P. Repetitive transcranial magnetic stimulation and chronic tinnitus. Acta Otolaryngol Supp/ 2006; (556): 102-5.

26. George MS, Wassermann EM, Post RM. Transcranial magnetic stimulation: a neuropsychiatric tool for the 21st century. J Neuropsychiatry Clin Neurosci 1996; 8: 373-82.

27. Langguth $B$, Zowe $M$, Landgrebe $M$, Sand $P$, Kleinjung T, Binder H, Hajak G, Eichnammer P. Transcranial magnetic stimulation for the treatment of tinnitus: a new coil positioning method and first results. Brain Topogr 2006; 18(4): 241-7.

28. Hoffman RE, Cavus I. Slow transcranial magnetic stimulation, long-term depotentiation, and brain hyperexcitability disorders. Am J Psychiatry 2002; 159: 1093-102.

29. Paus T, Castro-Alamancos MA, Petrides M. Corticocortical connectivity of the human mid- dorsolateral frontal cortex and its modulation by repetitive transcranial magnetic stimulation. Eur J Neurosci 2001; 14: 1405-11.

30. Baguley DM, Atlas MD. Cochlear implants and tinnitus. Prog Brain Res 2007; 166: 347-55.

31. Miyamoto RT, Bichey B. Cochlear implants for suppression of tinnitus. Otolaryngol Clin N Am 2003; 36: 345-52.

32. Quaranta N, WagstafF S, Baguley DM. Review paper. Tinnitus and cochlear implantation. Int $J$ Audiol 2004; 43: 245-51.

33. Quaranta N, Fernández-Vega S, D’Elia C, Filipo R, QuARANTA A. The effect of unilateral multichannel cochlear implant on bilaterally perceived tinnitus. Acta Oto-Laryngologica 2008; 128: 159-63.

34. Kleinjung T, Steffens T, Strutz J, LangGuth B. Curing tinnitus with a Cochlear Implant in a patient with unilateral sudden deafness: a case report. Cases J 2009; 2: 7462.

35. Moller AR. Pathophysiology of tinnitus. Otolaryngol Clin N Am 2003; 36: 249-66.

36. Van de Heyning P, Vermeire K, Diebl M, Nopp P, Anderson I, De Ridder D. Incapacitating, unilateral tinnitus in single sided deafness treated by cochlear implantation. Ann Otol Rhinol Laryngol 2008; 117: 645-52.

37. TYLER RS. Advantages and disadvantages expected and reported by cochlear implant patients. Am J Otol 1994; 15: 523-31.

38. Demajumdar R, Stoddart R, Donaldson I, Poops DW. Tinnitus, cochlear implants and how they affect patients. J Laryngol Otol Supp/ 1999; 24: 24-6.

39. McKerrow WS, Schreiner CE, Snyder RL, MerzenICH MM, TONer JG. Tinnitus suppression by cochlear implants. Ann Otol Rhinol Laryngol 1991; 100: 552-8.

40. Miyamoto RT, Wynne MK, McKnight C, Bichey B. Electrical suppression of tinnitus via cochlear implants. Int Tinnitus J 1997; 3: 35-8.

41. Souliere CR JR, Kileny PR, Zwolan TA, Kemink JL. Tinnitus suppression following cochlear implantation. A multifactorial investigation. Arch Otolaryngol Head Neck Surg 1992; 118: 1291-7.

42. Mo B, HarRIS S, LindBaek M. Tinnitus in cochlear implant patients: a comparison with other hearing impaired patients. Int J Audiol 2002; 41: 527-34. 
43. Ito J, Sakakihara J. Tinnitus suppression by electrical stimulation of the cochlear wall and by cochlear implantation. Laryngoscope 1994; 104: 752-4.

44. GIOLAS T. Aural rehabilitation of adults with hearing impairment; in Katz $\mathrm{J}$ (ed): Handbook of Clinical Audiology, ed 4. Baltimore, Williams \& Wilkins, 1994; 776-92.

45. Carhart R. Monaural and binaural discrimination against competing sentences. Intern Audiol 1965; 4: 5-10.

46. DIRKS DD, WILSON RH. The effect of spatially separated sound sources on speech intelligibility. J Speech Hear Res 1969; 12: 5-38.

47. MacKeith NW, Coles RRA. Binaural advantages in hearing of speech. $J$ Laryngol Otol 1971; 85 : 213-32.

48. Bronkhorst AW, Plomp R. The effect of headinduced interaural time and level differences on speech intelligibility in noise. $J$ Acoust Soc Am 1988; 83: 1508-16.

49. Vermeire K, Van de Heyning P. Binaural Hearing after Cochlear Implantation in Subjects with Unilateral Sensorineural Deafness and Tinnitus. Audiol Neurotol 2009; 14 : 163-71.
50. SHaw EA. Transformation of sound pressure level from the free field to the eardrum in the horizontal plane. J Acoust Soc Am 1974; 56: 1848-61.

51. Middlebrooks JC, GreEN DM. Sound localization by human listeners. Annu Rev Psychol 1991; 42: 135-59.

52. Arsenault MD, Punch JT. Nonsense-syllable recognition in noise using monaural and binaural listening strategies. J Acoust Soc Am 1999; 105: 1821-30.

53. Bronkhorst AW, Plomp R. A clinical test for the assessment of binaural speech perception in noise. Audiology 1990; 29: 275-85.

54. Dunn CC, TYLER R, WITT SA. Benefit of wearing a hearing aid on the unimplanted ear in adult users of a cochlear implant. J Speech Lang Hear Res 2005; 48: 668-80.

55. Ching TYC, Van Wankooy E, Hill M, Dillon H. Binaural redundancy and inter-aural time difference cues for patients wearing a cochlear implant and a hearing aid in opposite ears. Int $J$ Audiol 2005; 44: 677-90.

56. Ching TYC, Incerti P, Hill M, van Wanrooy E. An overview of binaural advantages for children and adults who use binaural/bimodal hearing devices. Audiol Neurotol 2006; 11(suppl 1): 6-11. 